\title{
Sterically Frustrated Aromatic Enes with Various Colors Originating from Multiple Folded and Twisted Conformations in Crystal Polymorphs
}

\author{
Tomohiko Nishiuchi, ${ }^{\text {[a] }}$ Seito Aibara, ${ }^{[a]}$ Takuya Yamakado ${ }^{[b]}$, Ryo Kimura ${ }^{[b]}$, Shohei Saito ${ }^{[b]}$, Hiroyasu \\ Sato ${ }^{[c]}$, and Takashi Kubo*[a,d]

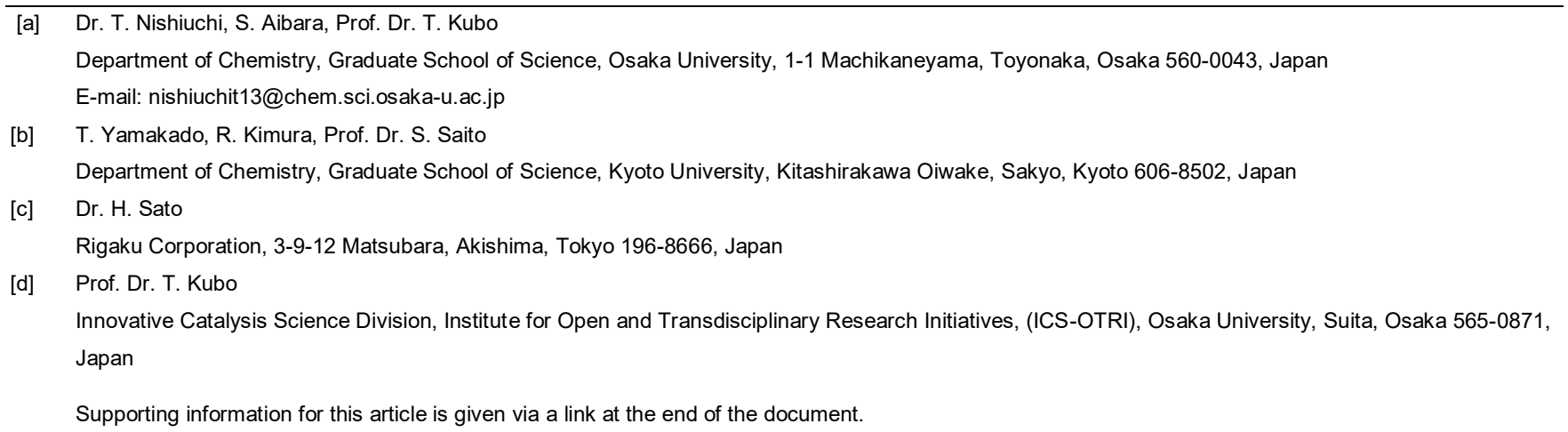

Abstract: Overcrowded ethylenes composed of 10methyleneanthrone and two bulky aromatic rings contain a twisted carbon-carbon double $(\mathrm{C}=\mathrm{C})$ bond as well as a folded anthrone unit. As such, they are unique frustrated aromatic enes (FAEs). Various colored crystals of these FAEs, obtained using different solvents, correspond to multiple metastable conformations of the FAEs with various twist and fold angles of the $\mathrm{C}=\mathrm{C}$ bond, as well as various dihedral angles of attached aryl units with respect to the $\mathrm{C}=\mathrm{C}$ bond. The relationships between color and these parameters associated with conformational features around the $\mathrm{C}=\mathrm{C}$ bond were elucidated using experimental and theoretical studies. Owing to the fact that they are separated by small energy barriers, the variously colored conformations in the FAE crystal change in response to various external stimuli, such as mechanical grinding, hydrostatic pressure and thermal heating.

\section{Introduction}

Chromic molecular materials that are stimulus response have attracted increasing interest due their applications in various light, heat, $\mathrm{pH}$, viscosity and mechanical stress sensors. ${ }^{[1]}$ In particular, flexible aromatic $\pi$-systems embedded with a movable component, such as cyclooctatetraene $(\mathrm{COT})^{[2,3]}$ or overcrowded ethylene (OCE) moiety, ${ }^{[4,5]}$ have been widely investigated. In these systems, conformations (bent or twisted) of the movable part govern the $\pi$-conjugation length of the molecule, which is directly reflected in optical properties (Figure 1). Therefore, to fine control of color change of the chromic behavior, it is necessary to have a properly designed potential energy surface associated with structural changes occurring in ground and excited states, that is, $S_{0}-S_{1}$ gap. For example, bianthorone is a typical OCE in which the ethylene group is part of two aromatic moieties and where steric hindrance is responsible for the existence of a yellow folded and a green twisted conformation. ${ }^{[6]}$ The calculated potential energy profile for interconversion between these conformations (Figure 2) suggests that the process like those of other OCEs has a high activation energy and that it takes place through no isolable intermediates. As a result, the bianthorone undergoes only a single color change between yellow and green in association with this conformational change. On the other hand, if the activation barriers separating several colored conformers are small, a continuous color change might be promoted by a slight energy input, provided by for example weak mechanical stress. In fact, COT based flexible $\pi$-systems exhibit this property in the form of a bend angle dependency of its optical properties. However, since small differences in the bent angle of the COT ring have little effect on the optical properties, it is still a challenging task to synthesize molecules whose optical properties are more sensitive to the slight structural changes. 
COT embedded flexible $\pi$-systems
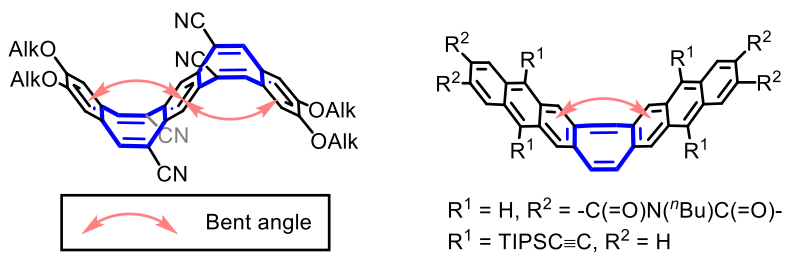

$\mathrm{R}^{1}=\mathrm{H}, \mathrm{R}^{2}=-\mathrm{C}(=\mathrm{O}) \mathrm{N}\left({ }^{n} \mathrm{Bu}\right) \mathrm{C}(=\mathrm{O})$ $\mathrm{R}^{1}=\mathrm{TIPSC} \equiv \mathrm{C}, \mathrm{R}^{2}=\mathrm{H}$

OCE embedded $\pi$-systems
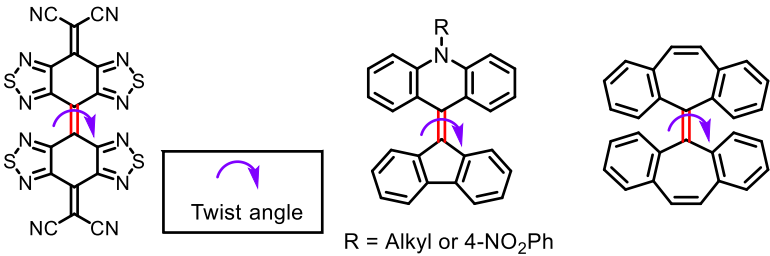

Figure 1. Typical flexible aromatic $\pi$-systems embedded within a COT or OCE unit.

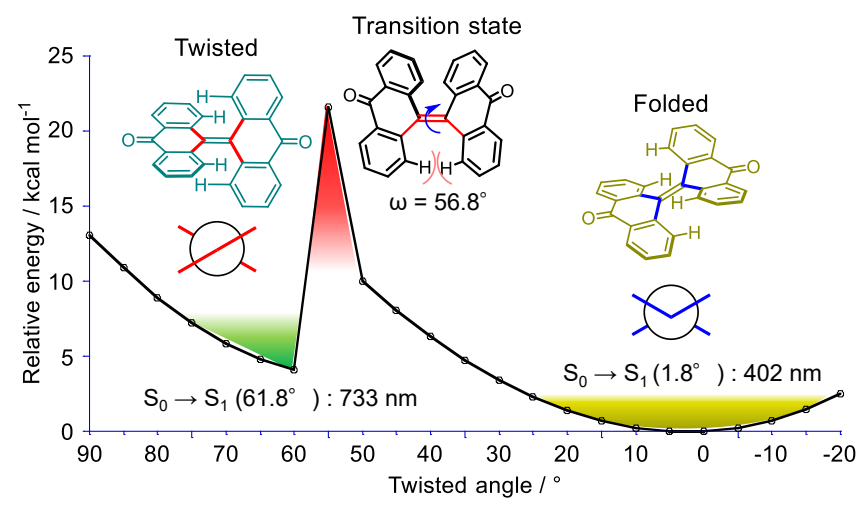

Figure 2. Folded and twisted conformations, and transition state structure of bianthrone and potential energies for every $5^{\circ}$ of twist angle $(\omega)$ together with the calculated $S_{0} \rightarrow S_{1}$ transitions of both the folded $\left(\omega=1.8^{\circ}\right)$ and twisted conformations $\left(\omega=61.8^{\circ}\right)$ calculated using RB3LYP/6-31G*.

The study described below was aimed at developing a strategy to finely control the conformational and optical properties of an OCE using an approach based on the incorporation of multiple moving components. The monotricyclic aromatic ene (MAE, Figure 3a) is known to exist in two conformations differing in the fold and twist of the carbon-carbon double $(\mathrm{C}=\mathrm{C})$ bond. The reported twist angle of the is substance is only $7.4^{\circ}$ (Figure $3 \mathrm{~b}$ ) and relationships between its conformation and properties, especially optical, have not been well studied. ${ }^{[7]}$ In the current effort, we designed two MAEs that contain a 10methyleneanthrone moiety and two appended bulky aromatic (9anthryl or mesityl) rings. The array of groups in these substances cause steric repulsion between the tricyclic anthrone unit and the two bulky aromatic rings, which leads to structural frustration of the $\mathrm{C}=\mathrm{C}$ bond associated with the formation of conformations having large fold and twist angles (Figure 3c). We have demonstrated that the sterically frustrated aromatic enes (FAEs) exist in multiple metastable conformations not only having a variety of different fold and twist angles about the $\mathrm{C}=\mathrm{C}$ bond but also different dihedral angles between the $\mathrm{C}=\mathrm{C}$ bond and the bulky aromatic rings. The various conformers have close energies in the solid state, and they exhibit unique absorption and emission colors. The relationships that exist between the properties of the conformers and the fold, twist and dihedral angles were elucidated by using experimental and theoretical studies.

(a) Monotricyclic Aromatic Ene (MAE)

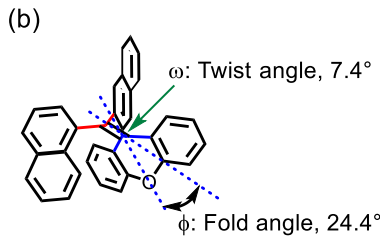

(c)

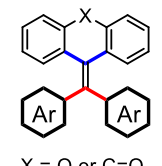

Folded/Twisted

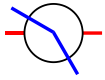

$\mathrm{X}=\mathrm{O}$ or $\mathrm{C}=\mathrm{O}$ $\phi$ : Fold angle, 24.4

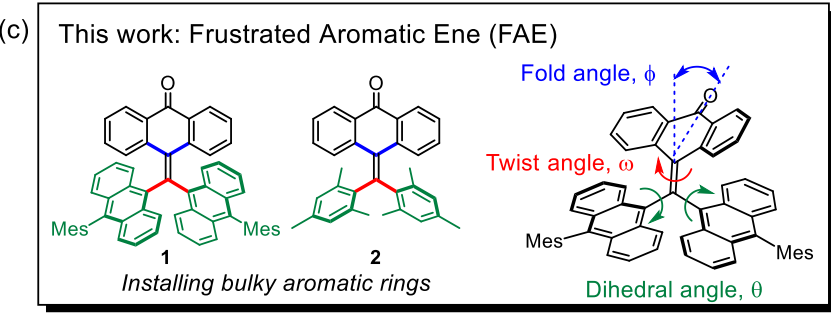

Figure 3. (a) The basic structure of monotricyclic aromatic ene (MAE) with its Newman projection to clarify the fold/twist angles of the $\mathrm{C}=\mathrm{C}$ bond. (b) First reported MAE composed of xanthene with two naphthyl rings and its fold angle $\Phi$ as well as twist angle $\omega$. (c) (left) FAE 1 and FAE 2 possessing a frustrated aromatic ene and (right) identifying twist angle $\omega$, fold angle $\varphi$, and dihedral angle $\theta$ (right)

\section{Results and Discussion}

\section{Conformation-Property Relationships of FAE in the Crystalline State}

The method employed to synthesize the bulky aromatic ring containing FAE 1, using Negishi coupling between 10(dibromomethylene)-9-anthrone and the substituted anthacenyl zinc chloride, was developed previously. ${ }^{\left[{ }^{8]}\right.}$ The analogous anthrone derivative FAE 2 was synthesized by using the same procedure (Scheme 1). A study exploring the optical properties of FAE 1 in solution showed that the absorption spectrum is only slightly changed by changing the solvent (Figure S1). FAE 1 was observed to form crystals of various colors in a variety of solvents (Figure 4a). Amber crystals were obtained from acetone (1-AO) or dichloromethane-hexane (1-DH), orange crystal were generated from DMF (1-DMF), red crystals were produced in hexafluorobenzene (1-6FB) or 1,1,2,2-tetrachloroethylene (1TCE), and dark red crystals were obtained from acetone (1-AD). $1-A D$ is a crystal polymorph of 1-AO. Due to the skeleton of the 
OCE, these crystals show aggregation-induced emission (AIE) behavior, ${ }^{[9]}$ and they display various emission colors of amber (1$\mathrm{AO}$ and 1-DH), orange (1-DMF), red (1-TCE and 1-6FB) and dark red (1-AD). The crystalline-state absorption and emission spectra of the crystals are shown in Figure $4 \mathrm{~b}-\mathrm{e}$ and key spectral data are listed in Table 1.
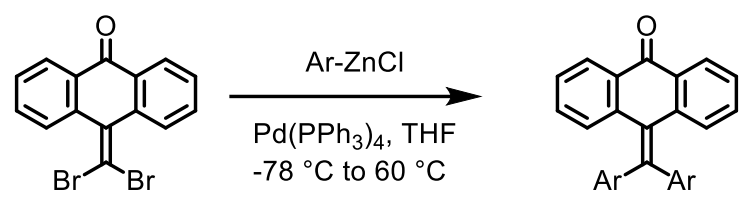

1: Ar = 10-mesityl-9-anthryl

2: $\mathrm{Ar}=$ mesityl

Scheme 1. Route for synthesis of FAE 1 and FAE 2.

Table 1. Absorption $\left(\lambda_{\mathrm{abs}}\right)$ and emission $\left(\lambda_{\mathrm{em}}\right)$ wavelengths, and emission quantum yields $\left(\Phi_{\mathrm{em}}\right)$ of 1-AO, 1-DH, 1-DMF, 1-TCE, 1-6FB and 1-AD.

\begin{tabular}{lccc}
\hline & $\lambda_{\text {abs }} / \mathrm{nm}^{[\mathrm{a}]}$ & $\lambda_{\mathrm{em}} / \mathrm{nm}^{[\mathrm{b}]}$ & $\Phi_{\mathrm{em}} / \%$ \\
\hline 1-AO & 562 & 585 & 23 \\
1-DH & 566 & 585 & 24 \\
1-DMF & 570 & 596 & 23 \\
1-TCE & 604 & 620 & 19 \\
1-6FB & 640 & 605 & 5 \\
1-AD & 657 & 615,650 (sh) & 2 \\
\hline [a] $\lambda_{\text {abs }}$ are onset of absorptions. [b] The $\lambda_{\text {em }}$ are peak maxima.
\end{tabular}

Even though 1-AO and 1-AD were both obtained from acetone their absorption and emission spectra are completely different. Notably, the difference between the absorption onsets $\left(\lambda_{\mathrm{abs}}\right)$ of 1-AO $\left(\lambda_{\mathrm{abs}}=562 \mathrm{~nm}\right)$ and 1-AD $\left(\lambda_{\mathrm{abs}}=657 \mathrm{~nm}\right)$ is $95 \mathrm{~nm}$ $\left(2573 \mathrm{~cm}^{-1}\right)$, which is the largest difference observed among the crystals of FAE 1 . The difference in emission maxima $\left(\lambda_{\mathrm{em}}\right)$ of these crystals of $65 \mathrm{~nm}\left(1710 \mathrm{~cm}^{-1}\right)$ is also the largest in the series. These finding indicate that the color difference between 1-AO and 1-AD does not originate from the polarity of recrystallization solvent. Finally, the emission quantum yields of all of the crystals of FAE 1 tend to decrease as their $\lambda_{a b s}$ and $\lambda_{e m}$ are red-shifted.

To gain information about the source(s) of the optical differences, the crystals were subjected to X-ray crystallographic analysis. The conformation of FAE 1 in 1-AO is shown in Figure 5. As expected, the presence of bulky 9-anthryl units in the FAE 1 skeleton leads not only a large fold angle for the $\mathrm{C}=\mathrm{C}$ bond $(\varphi$ $\left.=31.5^{\circ}\right)$ but also a large twist angle of the $\mathrm{C}=\mathrm{C}$ bond $\left(\omega=25.9^{\circ}\right)$.
Inspection of the crystallographic data for the other crystals (Figure S3-S7) showed that both the fold and twist angles vary as do the dihedral angles $\theta$ between the $C=C$ bond and two anthracenyl units. In addition, intermolecular interactions were found to exist between anthrone units in neighboring FAE 1 molecules in 1-AO. However, the interactions only occur at edge position with distances of $3.54 \AA(\mathrm{O} \cdots \mathrm{O})$ and $3.44 \AA(\mathrm{C} \cdots \mathrm{C})$ (Figure $5 \mathrm{c}$ ). Anthrone-anthrone interactions were also seen in the other crystals except 1-6FB, and the arrangement was different in each.

With the crystallographic data for the crystals of FAE 1 in hand, we next assessed whether a rational relationship exists between the conformational and optical properties. The key structural and conformational parameters, such as $\mathrm{C}=\mathrm{C}$ bond length, $\mathrm{C}=\mathrm{O}$ bond length in the anthrone unit, twist angle $\omega$ and fold angle $\varphi$ of the $C=C$ bond, and dihedral angle $\theta$ of 9-anthryl units are accumulated in Table 2. Although the $\mathrm{C}=\mathrm{C}$ and $\mathrm{C}=\mathrm{O}$ bond lengths were expected to depend on $\lambda_{\mathrm{abs}}$ and $\lambda_{\mathrm{em}}$ values for the crystals, a correlation between does not exist. IR peak positions for $\mathrm{C}=\mathrm{O}$ vibrations were determined and again no correlation was found between them and the optical properties (Figure S10).

Table 2. Parameters for conformational features around $C=C$ bond of $F A E$ 1. In each crystal: $C=C$ bond length $(\AA), C=O$ bond length $(\AA)$ in the anthrone unit, twist angle $\omega\left({ }^{\circ}\right)$ of $\mathrm{C}=\mathrm{C}$ bond, fold angle $\varphi\left({ }^{\circ}\right)$ of $\mathrm{C}=\mathrm{C}$ bond, and dihedral angle $\theta\left({ }^{\circ}\right)$ of anthracenyl units.

\begin{tabular}{lccccc}
\hline & $\mathrm{C}=\mathrm{C} / \AA$ & $\mathrm{C}=\mathrm{O} / \AA$ & $\omega /{ }^{\circ}$ & $\varphi /{ }^{\circ}$ & $\theta /{ }^{\circ}$ \\
\hline 1-AO & 1.379 & 1.222 & 25.9 & 31.5 & $53.6,67.7$ \\
1-DH & 1.365 & 1.224 & 26.3 & 30.7 & $53.4,66.1$ \\
1-DMF & 1.374 & 1.214 & 26.9 & 30.0 & $50.4,64.8$ \\
1-TCE & 1.380 & 1.227 & 27.3 & 30.7 & $53.0,62.0$ \\
1-6FB & 1.379 & 1.223 & 31.4 & 26.7 & $50.2,59.0$ \\
1-AD & 1.387 & 1.230 & 29.5 & 25.5 & $51.0,52.9$ \\
\hline
\end{tabular}

In contrast, we found that trends do exist in the $\omega, \varphi$ and $\theta$ values and that they correlate with the optical properties. Specifically, as the twist angle $\omega$ increases, the absorption onset and emission maximum shift to longer wavelength. Furthermore, the fold angles $\varphi$ for FAE 1 in the red and dark red crystals 1-6FB and $1-\mathrm{AD},\left(23.3^{\circ}, 26.7^{\circ}\right.$ and $25.5^{\circ}$, respectively) are smaller than those of the amber and orange crystals (1-AO: $31.5^{\circ}, 1-\mathrm{DH}: 30.7^{\circ}$, 1-DMF: $30.0^{\circ}$, and 1-TCE: $30.7^{\circ}$ ). 
(a)

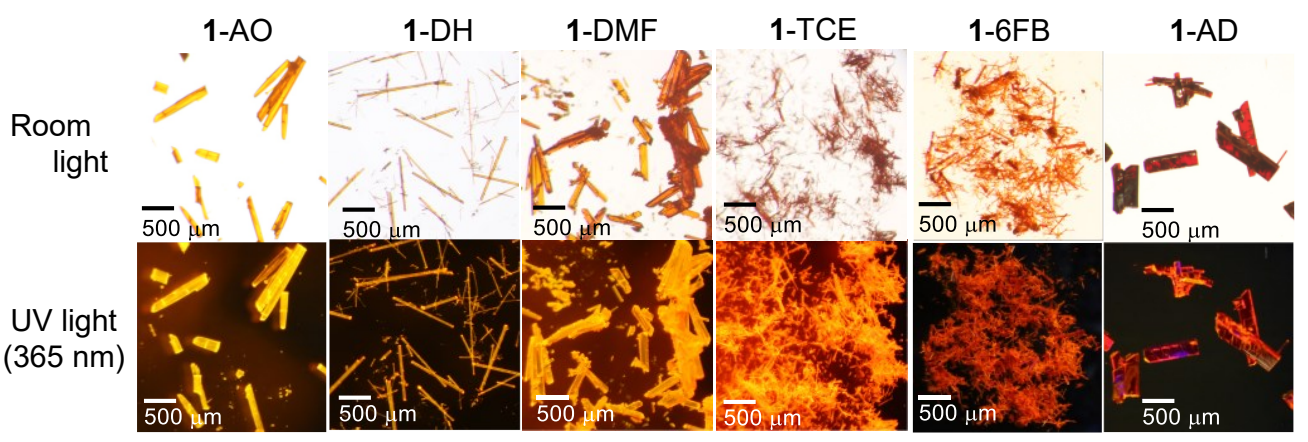

(b)

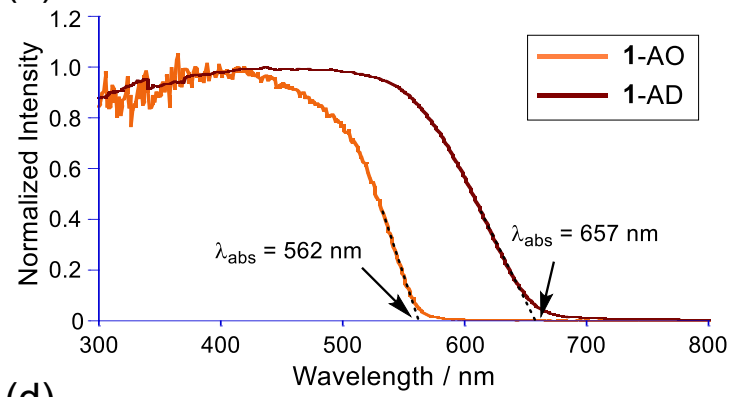

(d)

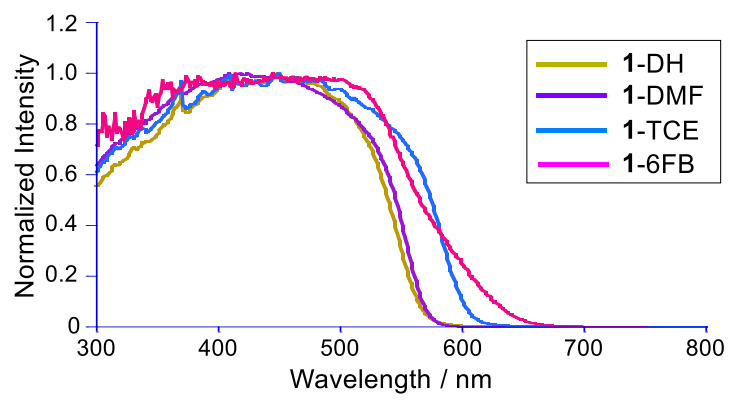

(c)

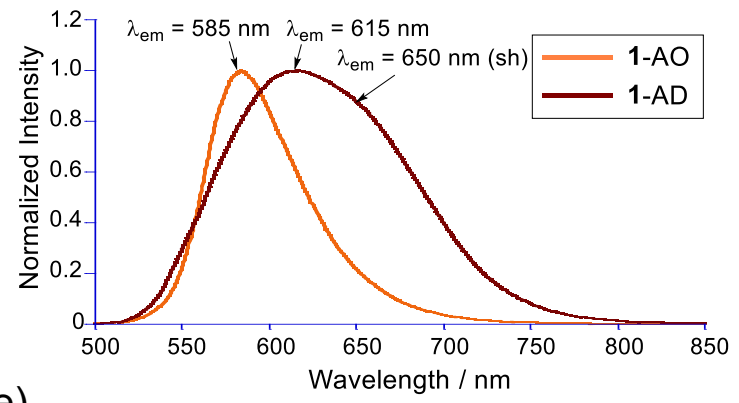

(e)

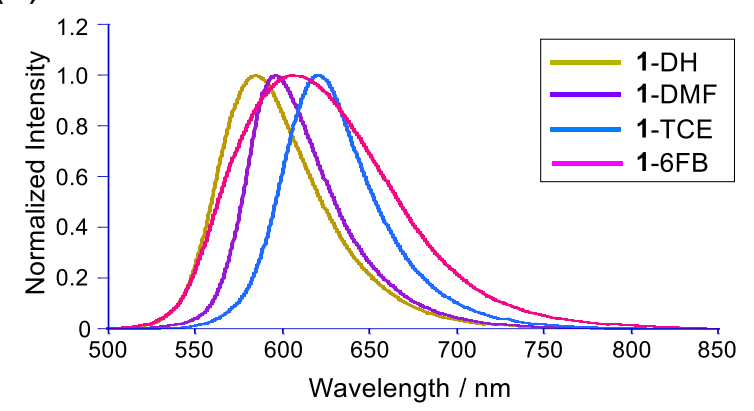

Figure 4. (a) Photoimages of various cyrtal colors of FAE 1 (1-AO, 1-DH, 1-DMF, 1-TCE, 1-6FB, and 1-AD, ) under room light and UV light (365 nm). (b) Crystalline-state diffuse-reflection absorption spectra of 1-AO and 1-AD. (c) Crystalline-state emission spectra of 1-AO and 1-AD. (d) Crystalline-state diffuse-reflection absorption spectra of 1-DH, 1-DMF, 1-TCE, and 1-6FB. (e) Crystalline-state emission spectra of 1-DH, 1-DMF, 1-TCE, and 1-6FB.

(a) Top view

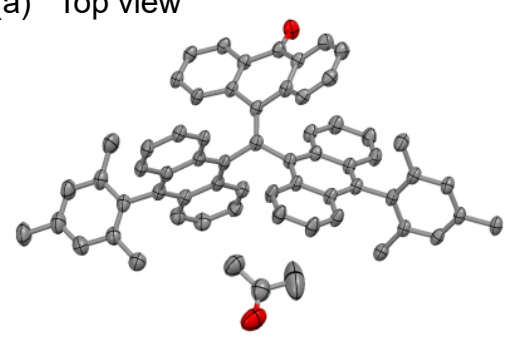

(b) Side view

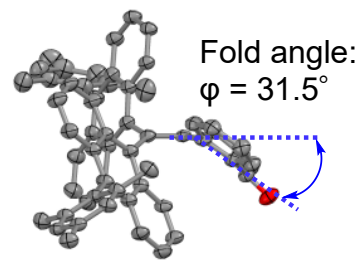

(c) Front view

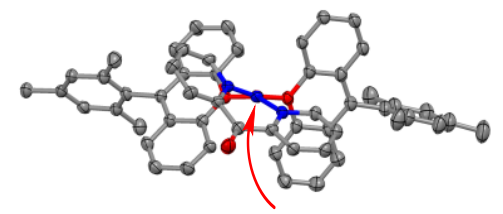

Twist angle: $\omega=25.9^{\circ}$

(d) Dimeric structure

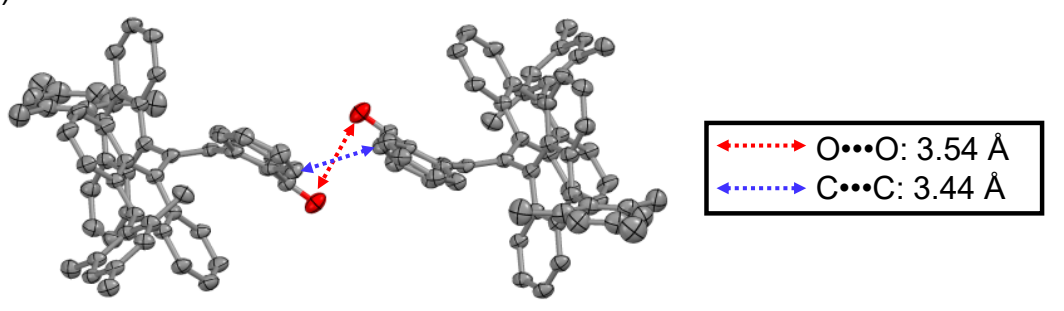

Figure 5. The molecular structure of FAE 1 in 1-AO. (a) Top view (together with a recrystallized aceton molecule). (b) Side view with folded angle of $\mathrm{C}=\mathrm{C}$ bond. (c) Front view with twisted angle of $\mathrm{C}=\mathrm{C}$ bond. (d) Intermolecular contact at the edge of anthrone unit with neighbored molecule. Protons are omitted for clarity. 
To gain more information about the relationship between these angles and the optical properties, TD-DFT calculations of conformations of FAE 1 with different twist and fold angles of the $\mathrm{C}=\mathrm{C}$ bond were conducted (Figure S12, S13). In Figure 6 is given a 3D map of TD-DFT results ( $x$-axis: twisted angle $\omega, y$-axis: folded angle $\varphi$, $z$-axis: $S_{0} \rightarrow S_{1}$ transition). Inspection of the map shows that a longer wavelength shift in the $S_{0} \rightarrow S_{1}$ transition takes place not only as the twist angle increases but also as the fold decreases. This finding is in good agreement with the experimental results. Within the range of the simulated fold and twist angles, distributions of the HOMO and LUMO orbitals of FAE 1 slightly expand with increasing twist angle, but they do not significantly change (Figure S14). It is noteworthy that calculated potential energy (PE) differences between the conformation corresponding to the shortest absorption wavelength at $517 \mathrm{~nm}$ $\left(\omega=25^{\circ}, \varphi=30^{\circ}\right)$ and to that of the longest wavelength at 637 $\mathrm{nm}\left(\omega=45^{\circ}, \varphi=15^{\circ}\right)$ is only $4.41 \mathrm{kcal} \mathrm{mol}^{-1}$ (Figure S15). In addition, the $\mathrm{PE}$ in $\mathrm{S}_{1}$ state is greatly stabilized by increasing twist angle and decreasing fold angle, showing the opposite trend to that in the $\mathrm{S}_{0}$ state (Figure $\mathrm{S} 16$ ). This finding suggests that a significant absorption wavelength change is triggered by only a slight conformational change in the solid state.

Analysis of the data indicates that dihedral angle $\theta$ is also an important factor governing optical properties. Comparison of the conformations of FAE 1 in crystals between 1-6FB and 1-AD shows that, although the absorption and emission properties are totally different, only a small difference exists in the twist and fold angles, but a significant difference is present in the dihedral angles. The dihedral angles $\theta$ of $1-6 \mathrm{FB}$ are $50.2^{\circ}$ and $59.0^{\circ}$, respectively, while those of $1-A D$ are $51.0^{\circ}$ and $52.9^{\circ}$. TD-DFT calculations indicate that decreasing the dihedral angle of FAE 1 , with fixed twist and fold angles leads to a longer wavelength shift in the absorption onset (Figure S17). Moreover, TD-DFT calculations on the monomer structures of FAE 1 in 1-6FB and 1$A D$ show that the $S_{0} \rightarrow S_{1}$ transition of 1-AD $(573 \mathrm{~nm})$ occurs at longer wavelength than that of 1-6FB $(531 \mathrm{~nm})$ (Table S1). Therefore, the optical differences between 1-6FB and 1-AD are mainly due to a difference in their dihedral angles.

The crystallographic data for 1-DMF and 1-TCE provide further information about factors that govern optical properties of $F A E$ 1. No significant differences exist in the $\omega, \varphi$ and $\theta$ values of 1-DMF and 1-TCE, yet their absorption and emission spectra differ. Inspection of the crystal packing structures shows that the anthrone unit in 1-TCE engages in significant intermolecular $\pi-\pi$ interaction with the anthrone unit of a neighboring molecule, while no anthrone-anthrone $\pi-\pi$ interaction occurs in 1-DMF (Figure S4, S5). TD-DFT calculations were carried out on monomeric and

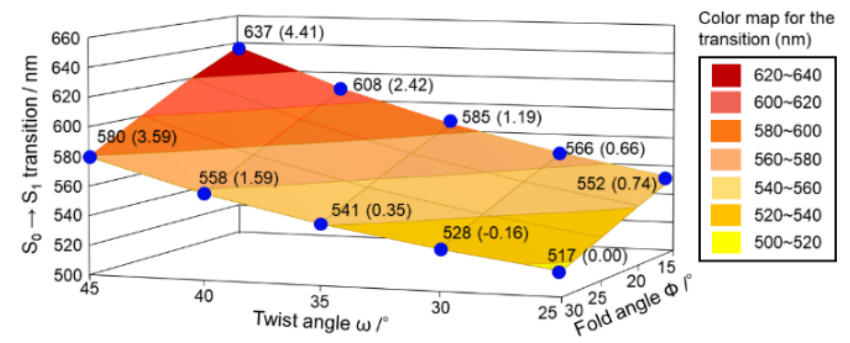

Figure 6. 3D map of TD-DFT calculated results (B3LYP/6-31G*//B3LYP/6$31 \mathrm{G}^{*}$ ) of FAE 1 ( $x$-axis: twist angle $\omega$ from $25^{\circ}$ to $45^{\circ}, y$-axis: fold angle $\varphi$ from $15^{\circ}$ to $30^{\circ}, z$-axis: $S_{0} \rightarrow S_{1}$ transition). The numbers near the blue dots on the $3 D$ map surface indicate the $S_{0} \rightarrow S_{1}$ transition at the position, and the numbers in parentheses indicate the relative potential energy at the position ( $\mathrm{kcal} \mathrm{mol}^{-1}$, the referring structure is $\omega=25^{\circ}, \varphi=30^{\circ}$, calculated by B3LYP/6-31G*)

dimeric structures. The results indicate that the $S_{0} \rightarrow S_{1}$ transition of the monomeric structure of 1-TCE occurs at $524 \mathrm{~nm}$, which is a longer wavelength than that of monomeric 1-DMF (509 nm). Moreover, the dimeric structure of 1-TCE, having large $\pi-\pi$ overlap between the anthrone units, has longer wavelength $\mathrm{S}_{0} \rightarrow$ $S_{1}$ transition at $531 \mathrm{~nm}$, whereas that of dimeric structure of 1 DMF is at $509 \mathrm{~nm}$ (Table S1). Thus, although the effect of the intermolecular $\pi-\pi$ interaction between the anthrone units cannot exclude as a factor affecting the color difference between1-DMF and 1-TCE, ${ }^{[10]}$ it can be concluded that the main contribution for the various colors in these crystals is the slight structural difference around the frustrated aromatic $\mathrm{C}=\mathrm{C}$ bond.

To gain further information, the optical properties of FAE 2 in the crystalline state were determined. Different from FAE 1, FAE 2 generates only two crystal polymorphs that do not contain co-cystallization solvent and exhibit yellow (2-Y) and orange (2O) colors (Figure 7a). The respective UV-vis and emission wavelengths of $2-Y$ are $\lambda_{\mathrm{abs}}=515 \mathrm{~nm}$ and $\lambda_{\mathrm{em}}=555 \mathrm{~nm}$ and it has a relatively high emission quantum yield of $32 \%$. In contrast, $2-O$ has $\lambda_{\mathrm{abs}}=560 \mathrm{~nm}$ and $\lambda_{\mathrm{em}}=610 \mathrm{~nm}$ along with a low emission quantum yield of $1 \%$ (Figure $7 \mathrm{~b}-\mathrm{c}$ ). X-ray crystallographic analysis shows that 2-O is comprised of two independent conformations 2 $\mathrm{O}-\mathrm{a}$ and $2-\mathrm{O}-\mathrm{b}$, while only one conformation exists for $2-\mathrm{Y}$ (Figure 8). The key parameters around the frustrated $\mathrm{C}=\mathrm{C}$ bond are listed in Table 3. TD-DFT calculations show that the $S_{0} \rightarrow S_{1}$ transition of 2-O-a is estimated to occur at $398 \mathrm{~nm}$, which is shorter than that of 2-Y $(411 \mathrm{~nm})$, owing likely to the existence of a large fold angle $\varphi$ in the latter (2-Y: 20.4 $\left.{ }^{\circ}, 2-\mathrm{O}-a: 29.7^{\circ}\right)$ (Figure S18, Table S2). On the other hand, 2-O- $b$ has a larger twist angle $\left(\omega=26.3^{\circ}\right)$, smaller fold angle $\left(\varphi=24.1^{\circ}\right)$ and smaller dihedral angles $(\theta=$ $51.6^{\circ}$ and $59.9^{\circ}$ ) than does 2-O-a. These factors cause the calculated $\mathrm{S}_{0} \rightarrow \mathrm{S}_{1}$ transition of 2-O-a to take place at $419 \mathrm{~nm}$. The experimental and theoretical results arising from studies of FAE 2 are consistent with those coming from FAE 1. Although an anthrone-anthrone interaction is present in 2-O, TD-DFT calculations show that its effect on the absorption wavelength is 
relatively small, which is identical to the tendency seen with FAE 1.
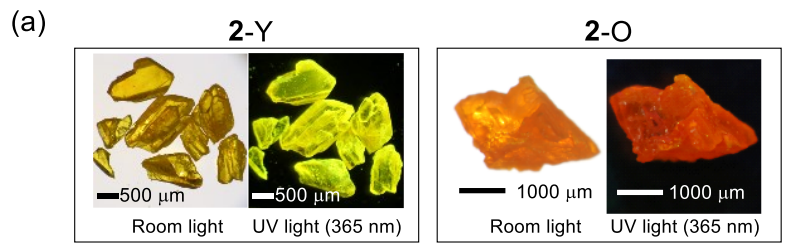

(b)

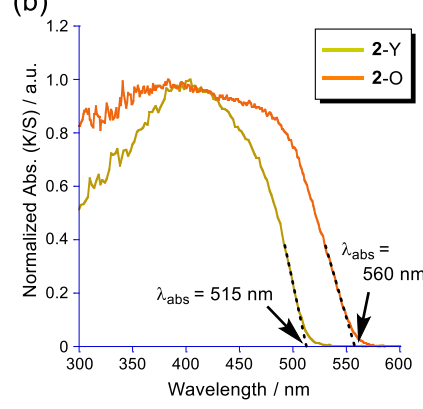

(c)

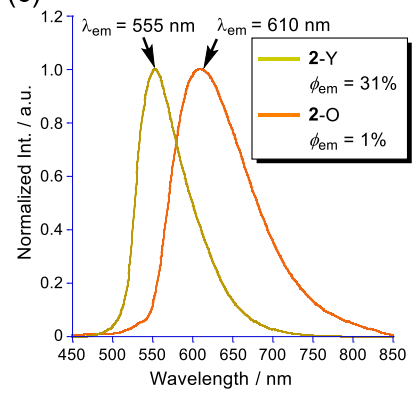

Figure 7. (a) Photoimages of the cyrstals 2-Y (left) and 2-O (right) under room light and UV light $(365 \mathrm{~nm})$. (b) Crystalline-state diffuse-reflection absorption spectra of 2-Y and 2-O. (c) Crystalline-state emission spectra and emission quantum yields of $2-Y$ and $2-O$. (a)

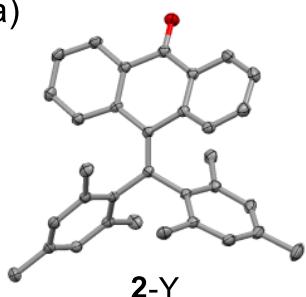

2-Y (b)

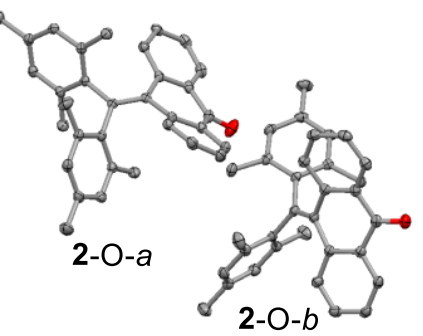

Figure 8. (a) Conformation of FAE 2 in 2-Y. (b) Conformation of FAE 2 in 2-O. Two independent conformations 2-O- $a$ and 2-O-b are observed. Protons are omitted for clarity.

Table 3. Parameters for conformational features around the $C=C$ bond of $F A E$ 2: $C=C$ bond length $(\AA), C=O$ bond length $(\AA)$ in the anthrone unit, twist angle $\omega$ $\left({ }^{\circ}\right)$ of $\mathrm{C}=\mathrm{C}$ bond, fold angle $\varphi\left({ }^{\circ}\right)$ of $\mathrm{C}=\mathrm{C}$ bond, and dihedral angle $\theta\left({ }^{\circ}\right)$ of anthracenyl units.

\begin{tabular}{lccccc}
\hline & $\mathrm{C}=\mathrm{C} / \AA$ & $\mathrm{C}=\mathrm{O} / \AA$ & $\omega /{ }^{\circ}$ & $\varphi /{ }^{\circ}$ & $\theta /{ }^{\circ}$ \\
\hline 2-Y & 1.377 & 1.230 & 19.3 & 20.4 & $57.8,62.2$ \\
$2-\mathrm{O}-\mathrm{a}$ & 1.370 & 1.223 & 22.4 & 29.7 & $51.5,63.5$ \\
2-O- $b$ & 1.380 & 1.231 & 26.3 & 24.1 & $51.6,59.9$ \\
\hline
\end{tabular}

\section{Mechano-, Piezo-, and Thermochromism of FAE skeleton}

Similar to those of other OCEs, FAE 1 and $\mathbf{2}$ display mechanochromic behavior in the solid state. ${ }^{[4-h, 11]}$ For example, grinding an orange crystal of FAE 1 or yellow crystal of FAE 2 causes the colors to change to dark red and orange color respectively (Figure 9, Figure S19). The color changes take place synchronously with a red-shift in the emission bands as well as a reduction in emission quantum yields (Figure 10). These changes are a consequence of alterations occurring in the parameters associated with conformational features surrounding the frustrated $\mathrm{C}=\mathrm{C}$ bond as was discussed above.

Because crystal 2-Y has a large crystal size and it does not contain a co-crystallization solvent, it is suitable for investigating how structural changes occur under application of hydrostatic pressure. ${ }^{[12]}$ When hydrostatic pressure was applied to $2-Y$ using a diamond anvil cell (DAC), the crystal color changed from yellow to amber (Figure 11a). The emission maximum underwent a corresponding red-shift from $555 \mathrm{~nm}$ to $565 \mathrm{~nm}$ (Figure 11b, Figure S20), which is similar to the change taking place when it is ground. Single crystal X-ray measurements made after applying hydrostatic pressure at $3.5 \mathrm{GPa}$ revealed that the color change does not originate from an increase in twist angle $\omega$ but rather to flattening of the fold angle $\varphi$ (Figure 11c, Figure S21). Specifically, the twist angle $\omega$ decreases from $20.0^{\circ}$ to $15.8^{\circ}$ and the fold angle $\varphi$ also decreases from $21.1^{\circ}$ to $12.4^{\circ}$. TD-DFT calculations support the proposal that this conformational change leads to a red-shift of the $S_{0} \rightarrow S_{1}$ transition from $392 \mathrm{~nm}(0 \mathrm{GPa})$ to $400 \mathrm{~nm}$ (3.5 GPa). Thus, this result indicates that the red-shift associated mechanochromism of FAE 1 and $\mathbf{2}$ is not solely due to an increase in the twist angle of the $\mathrm{C}=\mathrm{C}$ bond
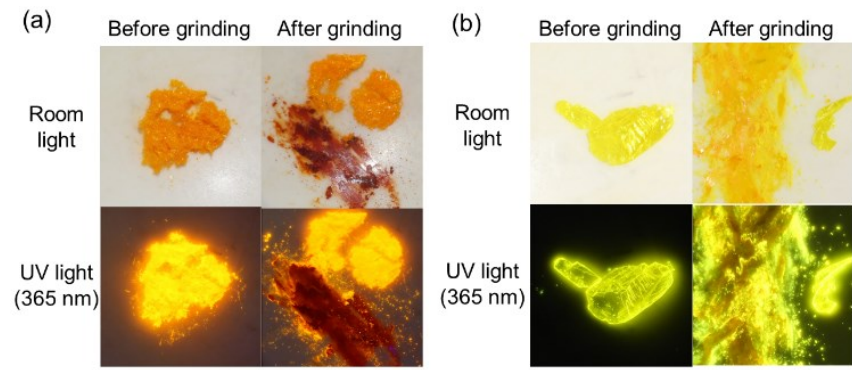

Figure 9. Images before and after grinding (a) 1-DH and (b) 2-Y under room and UV light (365 $\mathrm{nm})$.
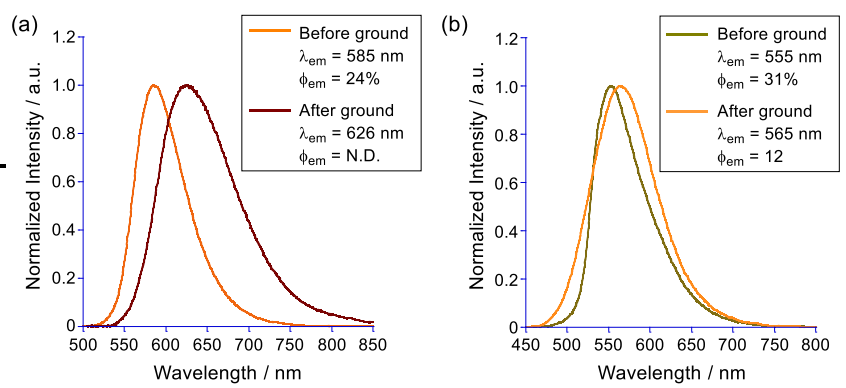

Figure 10. (a) Emission spectra before and after grinding 1-DH. (b) Emission spectra of before and after grinding 2-Y 
(a)

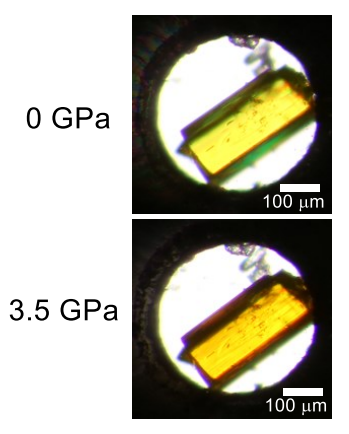

(b)

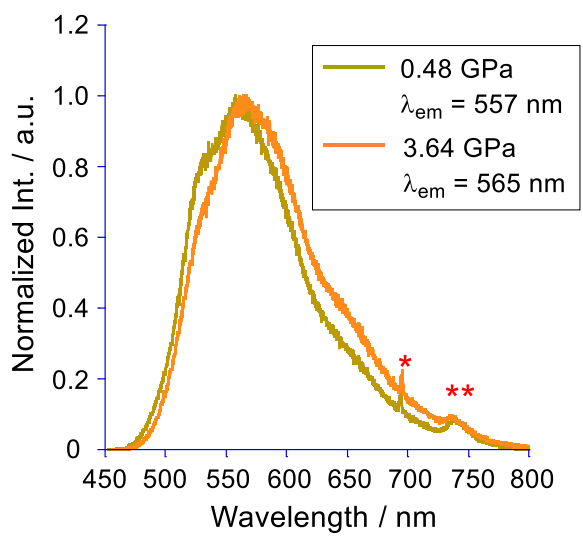

(c)

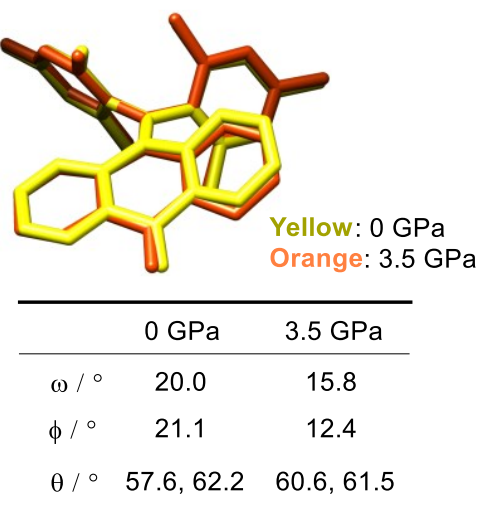

Figure 11. (a) Photoimages of 2-Y before (top) and after applying hydrostatic pressure with $3.5 \mathrm{GPa}$ (down). (b) Emission spectra of 2-Y under 0.48 GPa (yellow line) and $3.64 \mathrm{GPa}$ (orange line). Red ${ }^{*}$ indicates the emission from ruby. Red ${ }^{* *}$ indicates the second-order diffraction of excitation light $\left(\lambda_{\mathrm{ex}}=370\right.$ $\mathrm{nm})$. (c) Superposition of the X-ray crystalographic structures of 2-Y generated by before (0 GPa: yellow) and after applying hydrostatic pressure (3.5 GPa: orange).

(a)

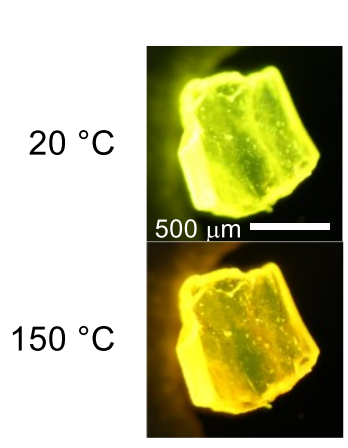

(b)

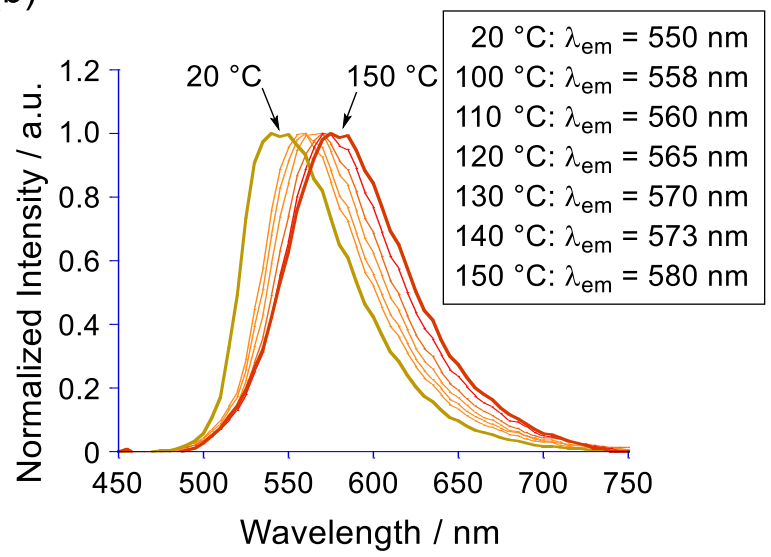

(c)

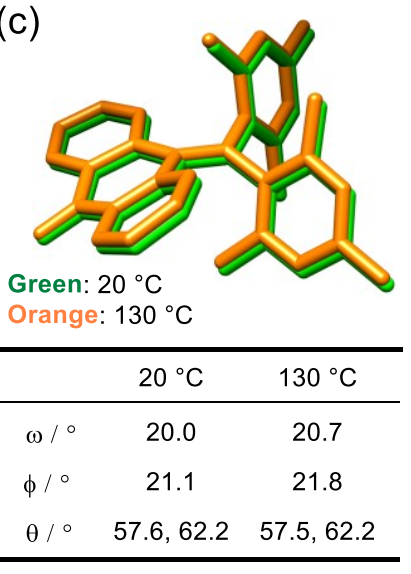

Figure 12. (a) Photoimages of $2-\mathrm{Y}$ at $20^{\circ} \mathrm{C}$ (top) and $150{ }^{\circ} \mathrm{C}$ (down) under UV light $\left(365 \mathrm{~nm}\right.$ ). (b) Emission spectra of $2-\mathrm{Y}$ at $20^{\circ} \mathrm{C}$ (yellow) to $150{ }^{\circ} \mathrm{C}$ (dark red) and emission peak tops $\lambda_{\mathrm{em}}$ at each temperature. (c) Superposition of the X-ray crystalographic structures of $2-\mathrm{Y}$ at $20^{\circ} \mathrm{C}$ (green) and $130{ }^{\circ} \mathrm{C}$ (orange).

Further efforts showed that crystal 2-Y exhibits thermochromic fluorescence behavior as well. ${ }^{[2 b, 13]}$ Upon heating 2-Y over $100{ }^{\circ} \mathrm{C}$, the fluorescence color gradually changes from yellow to orange and returned to yellow upon cooling (Figure 12a, Figure S22). The emission peak $\lambda_{\mathrm{em}}=550 \mathrm{~nm}$ at $20^{\circ} \mathrm{C}$ is clearly red-shifted to $580 \mathrm{~nm}$ at $150^{\circ} \mathrm{C}$ (Figure $12 \mathrm{~b}$ ), and the half-width of the emission band increases with an increase in temperature from $20{ }^{\circ} \mathrm{C}\left(520 \sim 590 \mathrm{~nm}, 2280 \mathrm{~cm}^{-1}\right)$ to $150{ }^{\circ} \mathrm{C}(545 \sim 625 \mathrm{~nm}, 2350 \mathrm{~cm}-$ $\left.{ }^{1}\right)$. Interestingly, a comparison of the $\mathrm{X}$-ray crystallographic data for $2-Y$ at $20{ }^{\circ} \mathrm{C}$ and $130{ }^{\circ} \mathrm{C}^{[14]}$ (Figure 12c) shows that no significant changes occur in the parameters related to conformation features around the frustrated $\mathrm{C}=\mathrm{C}$ bond. Rather, only a slight elongation of intermolecular distances takes place by heating, which results in an expansion of the void volume in a unit cell from $330 \AA^{3}$ to $386 \AA^{3}$ (Figure S23). ${ }^{[15]}$

TD-DFT calculations were conducted to estimate the lowest energy excited state conformation of FAE 2. The results show that the excited state of FAE 2 has a propeller shape with the twist angle $\omega=59.5^{\circ}$, fold angle $\varphi=0^{\circ}$ and dihedral angle $\theta=40.7^{\circ}$
(Figure S24). Therefore, a plausible explanation for the thermochromic fluorescence behavior of 2-Y associated with redshifted emission at high temperatures is that structural relaxation occurs through reduction of intermolecular interactions and expansion of the void volume to accommodate the twisted structure of the excited state. ${ }^{[16]}$ Furthermore, the lack of thermochromic fluorescence behavior of 2-O even though an identical tendency to expand the void volume in a unit cell occurs at high temperature (Figure S25,S26, Table S3). The difference is probably a consequence of intermolecular anthrone-anthrone interactions present 2-O that interfere with conformational relaxation enabling accommodation of the twisted structure of the excited state.

\section{Conclusion}

In the investigation described above, information was gained about relationships that exist between the conformational and optical properties of the crystal states of sterically frustrated 
aromatic enes (FAEs). We demonstrated experimentally and theoretically that FAE 1 and FAE 2 form various color crystals, that correspond to multiple metastable conformations with various twist and fold angles of the $\mathrm{C}=\mathrm{C}$ bond, as well as various dihedral angles of attached aryl units with the $\mathrm{C}=\mathrm{C}$ bond. Different from usual OCEs exhibiting only folded and twisted forms separated by large energy gaps and/or large activation energies, the FAEs possess conformational energy profiles in which multi-colored crystal polymorph with a variety of different fold, twist and dihedral angles exist as metastable intermediates. In addition, conformational/color changes of the FAE crystals can be readily induced by external stimuli such as mechanical grinding, hydrostatic pressure and thermal heating. We anticipate that the multi-chromic properties associated with slight conformational changes make the FAEs appropriate candidates for multipurpose sensors that can be utilized to detect small energy loads in artificial or biological materials. ${ }^{[17]}$

\section{Acknowledgements}

This study was supported by JSPS KAKENHI Grant-in-Aid for Scientific Research (C) JP20K05475 (T.N.), Transformative Research Areas (A) JP20H05865 (T.K.), and JP21H05482 (S.S.). Quantum chemical calculations were performed at the Research Center for Computational Science, Okazaki, Japan. T. N. thank Prof. Katsuya Shimizu (Centre for Science and Technology under Extreme Conditions, Graduate School of Engineering Science, Osaka University) for helping the setup of DAC, Prof. Takumi Konno and Prof. Nobuto Yoshinari (Graduate School of Science, Osaka University) for measurements of emission quantum yields, and Prof. Shuichi Suzuki (Graduate School of Engineering Science, Osaka University) for acquisition of HR-MS spectra.

Keywords: overcrowded ethylene - Metastable structures • Mechanochromism • Piezochromism • Thermochromism

[1] Typical reviews for chromic molecules, see: a) C. Reichardt, Chem. Soc. Rev. 1992, 21, 147.; b) E. Hadjoudis, I. M. Mavridis, Chem. Soc. Rev. 2004, 33, 579.; b) M. Irie, T. Fukaminato, K. Matsuda, S. Kobatake, Chem. Rev. 2014, 114, 12174.; c) D. Bléger, S. Hecht, Angew. Chem. Int. Ed. 2015, 54, 11338.; d) A. Fihey, A. Perrier, W. R. Browne, D. Jacquemin, Chem. Soc. Rev. 2015, 44, 3719.; d) M. A. Haidekker, E. A. Theodorakis, J. Mater. Chem. C. 2016, 4, 2707.; e) M. K. Kuimova, Phys. Chem. Chem. Phys. 2012, 14, 12671.; f) Z. Yang, J. Cao, Y. He, J. H. Yang, T. Kim, X. Peng, J. S. Kim, Chem.Soc. Rev. 2014, 43, 4563.; g) T. Kowada, H. Maeda, K. Kikuchi, Chem. Soc. Rev, 2015, 44, 4953.

[2] a) T. Nishiuchi, Y. Kuwatani, T. Nishinaga, M. Iyoda, Chem. Eur. J. 2009, 15, 6838.; b) T. Nishiuchi, K. Tanaka, Y. Kuwatani, J. Sung, T. Nishinaga, D. Kim, M. Iyoda, Chem. Eur. J. 2013, 19, 4110.; c) T. Nishiuchi, M. Iyoda,
Bull. Chem. Soc. Jpn. 2014, 87, 960; d) T. Nishiuchi, M. Iyoda, Chem. Rec. 2015, 15, 329.

[3] a) C. Yuan, S. Saito, C. Camacho, S. Irle, I. Hisaki, S. Yamaguchi, J. Am Chem. Soc. 2013, 135, 8842; b) C. Yuan, S. Saito, C. Camacho, T. Kowalczyk, S. Irle, S. Yamaguchi, Chem. Eur. J. 2014, 20, 2193.; c) S. Saito, S. Nobusue, E. Tsuzaka, C. Yuan, C. Mori, M. Hara, T. Seki, C Camacho, S. Irle, S. Yamaguchi, Nat. Commun. 2016, 7, 12094.; d) R. Kotani, H. Sotome, H. Okajima, S. Yokoyama, Y. Nakaike, A. Kashiwagi, C. Mori, Y. Nakada, S. Yamaguchi, A. Otsuka, A. Sakamoto, H. Miyasaka, S. Saito, J. Mater. Chem. C. 2017, 5, 5248.; e) T. Yamakado, K. Otsubo, A. Osuka, S. Saito, J. Am. Chem. Soc. 2018, 140, 6245.; f) R. Kimura, H. Kuramochi, P. Liu, T. Yamakado, A. Osuka, T. Tahara, S. Saito, Angew. Chem. Int. Ed. 2020, 59, 16430.

[4] Typical examples of OCEs composed of bistricyclic aromatic ene skeleton, see: a) I. Agranat, M. R. Suissa, Struct. Chem. 1993, 4, 59.; b) T. Suzuki, T. Fukushima, T. Miyashi, T. Tsuji, Angew. Chem. Int. Ed. Engl. 1997, 36, 2495.; c) P. U. Biedermann, J. J. Stezowski, I. Agranate, Chem. Eur. J. 2006, 12, 3345.; d) C. Wentrup, M. J. Regimbald-Krnel, D. Müller, P. Comba, Angew. Chem. Int. Ed. 2016, 55, 14600.;.e) A. Takai, D. J. Freas, T. Suzuki, M. Sugimoto, J. Labuta, R. Haruki, R. Kumai, S-i. Aadachi, H. Sakai, T. Hasobe, Y. Matsushita, M. Takeuchi, Org. Chem. Front. 2017, 4, 650.; f) T. Suzuki, H. Okada, T. Nakagawa, K. Komatsu, C. Fujimoto, H. Kagi, Y. Matsuo, Chem. Sci. 2018, 9, 475.; g) Y. Matsuo, Y. Wang, H. Ueno, T. Nakagawa, H. Okada, Angew. Chem. Int. Ed. 2019, 58, 8762.; h) Y. Wang, Y. Ma, K. Ogumi, B. Wang, T. Nakagawa, Y. Fu, Y. Matsuo, Commun. Chem. 2020, 3, 93.; i) Y. Adachi, T. Nomura, S Tazuhara, H. Naito, J. Ohshita, Chem. Commun. 2021, 57, 1316.

[5] Typical examples of recent OCEs composed of anthraquinodimethane core, see: a) X. Zhang, X. Jiang, K. Zhang, L. Mao, J. Luo, C. Chi, H. S. O. Chan, J. Wu, J. Org. Chem. 2010, 75, 8069.; b) T. Suzuki, Y. Ishigaki, K. Sugawara, Y. Umezawa, R. Katoono, A. Shimoyama, Y. Manabe, K. Fukase, T. Fukushima, Tetrahedron 2018, 74, 2239.; c) X. Yin, J. Z. Low, K. J. Fallon, D. W. Paley, L. M. Campos, Chem. Sci. 2019, 10, 10733.; d) Y. Ishigaki, Y. Hayashi, T. Suzuki, J. Am. Chem. Soc. 2019, 141, 18293.; e) Y. Ishigaki, T. Hashimoto, K. Sugawara, S. Suzuki, T. Suzuki, Angew. Chem. Int. Ed. 2020, 59, 6581.; f) T. Nishiuchi, R. Ito, E. Stratmann, T. Kubo, J. Org. Chem. 2020, 85, 179.; g) K. Li, Z. Xu, J. Xu, T. Weng, X. Chen, S. Sato, J. Wu, Z. Sun, J. Am. Chem. Soc. 2021, 143 , 20419.

[6] Typical examples for bianthrones, see: a) $\mathrm{H}$. Meyer, Ber. Dtsch. Chem. Ges. B. 1909, 42, 143.; b) H. Meyer, Monatsh. Chem. 1909, 30, 165.; c) Y. Taouhi, O. Kalisky, I. Agranat, J. Org. Chem. 1979, 44, 1949.; d) Y. Hirao, Y. Hamamoto, N. Nagamachi, T. Kubo, Phys. Chem. Chem. Phys. 2019, 21, 12209.; e) Y. Hamamoto, Y. Hirao, T. Kubo, J. Phys. Chem. Lett. 2021, 12, 4729.

[7] a) N. Assadi, S. Pogodin, S. Cohen, I. Agranat, Struct. Chem. 2015, 26, 319.; b) N. Assadi, S. Pogodin, S. Cohen, I. Agranat, Asian. J. Org. Chem. 2015, 4, 1392

[8] T. Nishiuchi, S. Aibara, T. Kubo, Angew. Chem. Int. Ed. 2018, 57, 16516.

[9] Typical reviews for aggregation-induced emission, see: a) J. Mei, N. L. C. Leung, R. T. K. Kwok, J.W. Y. Lam, B. Z. Tang, Chem. Rev. 2015, 115, 11718.; b) Y. Xie, Z. Li, Chem. Asian. J. 2019, 14, 2524.; c) Z. Zhao, H. Zhang, J. W. Y. Lam, B. Z. Tang, Angew. Chem. Int. Ed. 2020, 59, 9888.; d) X. Cai, B. Liu, Angew. Chem. Int. Ed. 2020, 59, 9868.

[10] Although 1-AD also has an anthrone-anthrone $\pi-\pi$ interaction, the magnitude of the longer wavelength shift in the $S_{0} \rightarrow S_{1}$ transition (Monomer: $573 \mathrm{~nm}$, Dimer: $578 \mathrm{~nm}$ ) is smaller than that of 1-TCE. 
[11] Examples of recent mechanochromic molecules, see; a) $H$. Ito, $M$. Muromoto, S. Kurenuma, S. Ishizuka, N. Kitamura, H. Sato, T. Seki, Nat Commun. 2013, 4, 2009.; b) T. Seki, K. Sakurada, H. Ito. Angew. Chem. Int. Ed. 2013, 52, 12828.; c) S. Suzuki, R. Maya, Y. Uchida, T. Naota, ACS Omega, 2019, 4, 10031.; d) Y. Ishigaki, K. Sugawara, M. Yoshida, M. Kato, T. Suzuki, Bull. Chem. Soc. Jpn. 2019, 92, 1211.; e) T. Nishiuchi, K. Kisaka, T. Kubo, Angew. Chem. Int. Ed. 2020, 60, 5400.; f) Y. Tani, M. Komura, T. Ogawa, Chem. Commun. 2020, 56, 6810.

[12] Examples of hydrostatic pressure induced chromism, see: a) K. Nagura, S. Saito, H. Yusa, H. Yamawaki, H. Fujihisa, H. Sato, Y. Shimoikeda, S. Yamaguchi, J. Am. Chem. Soc. 2013, 135, 10322.; b) Q. Sui, X. -T. Ren, Y. -Z. Dai, K. Wang, W. -T. Li, T. Gong, J. -J. Fang, B. Zou, E. -Q. Gao, L. Wang, Chem. Sci. 2017, 8, 2758.; c) B. Yu, Y. Wang, L. Wang, X. Tan, Y. -M. Zhang, K. Wang, M. Li, B. Zou, S. X. -A. Zhang, Phys. Chem. Chem. Phys. 2019, 21, 17696.; d) Q. Sui, P. Li, R. Sun, Y. -H. Fang, L. Wang, B. -W. Wang, E. -Q. Gao, S. Gao, J. Phys. Chem. Lett. 2020, 11, 9282.; e) D. Zhao, M. Wang, G. Xiao, B. Zou, J. Phys. Chem. Lett. 2020 11, 7297.

[13] Examples of reversible thermochromic molecules, see; a) Y. Morita, S. Suzuki, K. Fukui, S. Nakazawa, H. Kitagawa, H. Kishida, H. Okamoto, A. Naito, A. Sekine, Y. Ohashi, M. Shiro, K. Sasaki, K. Sato, T. Takui, K. Nakasuji, Nat. Mater. 2008, 7, 48.; b) S. Perruchas, C. Tard, X. F. L. Goff, A. Fargues, A. Garcia, S. Kahlal, J. -Y. Saillard, T. Gacoin, J. -P. Boilot, Inorg. Chem. 2011, 50, 10682.; c) K. Shimada, A. Kobayashi, Y. Ono, H. Ohara, T. Hasegawa, T. Taketsugu, E. Sakuda, S. Akagi, N. Kitamura, M. Kato, J. Phys. Chem. C. 2016, 120, 16002.; d) T. Nishiuchi, H. Sotome, R. Fukuuchi, K. Kamada, H. Miyasaka, T. Kubo, Aggregate, 2021, 2, e126.

[14] Upon heating 2-Y from 140 to $160^{\circ} \mathrm{C}$ below the melting point ( $\mathrm{mp}$ : 163 $165^{\circ} \mathrm{C}$ ) and then standing for several minutes, a single-crystal-to-singlecrystal (SCSC) transition to 2-O begins (Figure S27). Thus, X-ray crystallographic analysis of $2-Y$ over $140{ }^{\circ} \mathrm{C}$ could not be performed. This thermal induced SCSC transition is a known phenomenon in several organic compounds, see; a) F. H. Herbstein, Acta. Crystallogr. Sect. B. Struct. Sci. 2006, 62, 341.; b) X. Luo, J. Li, C. Li, L. Heng, Y. Q. Dong, Z. Liu, Z. Bo, B. Z. Tang, Adv. Mater. 2011, 23, 3261.; c) Y. Abe, S. Karasawa, N. Koga, Chem. Eur. J. 2012, 18, 15038.

[15] Crystal void volume in a unit cell was calculated by CrystalExplorer program (version 21.5), P. R. Spackman, M. J. Turner, J. J. McKinnon, S. K. Wolff, D. J. Grimwood, D. Jayatilaka, M. A. Spackman, J. Appl. Cryst. 2021, 54, 1006.

[16] Reversible SCSC transition accompanied with thermochromic fluorescence behaviour was reported, see; S. Yang, P.-A. Yin, L, Li, Q. Peng, X. Gu, G. Gao, J. You, B. Z. Tang, Angew. Chem. Int. Ed. 2020, 59, 10136.

[17] Examples of force-responsive materials, see; (a) M. K. Beyer, H. C-. Schaumann, Chem. Rev. 2005, 105, 2921.; (b) M. M. Caruso, D. A. Davis Q. Shen, S. A. Odom, N. R. Sottos, S. R. White, J. S. Moore, Chem. Rev. 2009, 109, 5755.; (c) A. L. Black, J. M. Lenhardt, S. L. Craig, J. Mater. Chem. 2011, 21, 1655.; (d) J. R-. Arino, D. Mark, Chem. Rev. 2012, 112, 5412.; (e) J. Li, C. Nagamani, J. S. Moore, Acc. Chem. Res. 2015, 48, 2181.; (f) Y. Liu, K. Galior, V. P.. Y. Ma, K. Salaita, Acc. Chem. Res. 2017 50, 2915.; (g) N. W-. Fox, E. Rognin, T. A. Aljohani, R. Daly, Chem 2018, 4, 2499.; (h) J. M. Brockman, A. T. Blanchard, V. P-. Y. Ma, W. D. Derricotte, Y. Zhang, M. E. Fay, W. A. Lam, F. A. Evangelista, A. L. Mattheyses, K. Salaita, Nat. Methods 2018, 15, 115.; (i) R. Kotani, S.
Yokoyama, S. Nobusue, S. Yamaguchi, A. Osuka, H. Yabu, S. Saito, Nat. Commun. 2022, 13, 303 